\title{
Nephrotoxicity of cancer therapeutic drugs: Focusing on novel agents
}

\section{Min Hee Hong}

Division of Medical Oncology, Department of Internal Medicine, Yonsei Cancer Center, Severance Hospital, Yonsei University College of Medicine, Seoul, Republic of Korea

Kidney injury caused by anticancer agents is a common problem that can interfere with and affect the dose intensity of anticancer therapy, thus restricting patient survival. Recent advances in targeted and immunotherapeutic agents have transformed the landscape of medical oncology, and these agents have been widely employed in clinical practice. While typically associated with favorable toxicity profiles, several novel anticancer drugs present distinctive nephrotoxicities. It remains urgent to closely monitor renal injuries associated with these agents, and medical practitioners should be familiar with general principles for managing nephrotoxicity associated with novel cancer drugs. This review provides an in-depth investigation of the literature and guidelines regarding the prevalence, clinical presentations, mechanisms, and management of nephrotoxicity for each drug.

Keywords: Acute kidney injury, Immune checkpoint inhibitors, Molecularly targeted therapy, Neoplasms, Renal insufficiency

\section{Introduction}

In recent decades, advances in cancer chemotherapeutics have improved both the survival and quality of life of patients with cancer. Novel chemotherapeutic agents, such as targeted molecular agents and immunotherapeutics, have been developed and widely adopted in clinical practice $[1,2]$. Compared to cytotoxic agents, these drugs present fewer conventional adverse events such as alopecia, nausea/ vomiting, fatigue, and bone marrow suppression. However, drug-induced kidney injury remains a frequent challenge.
A variety of renal complications can occur among patients with malignancy, and these drugs can affect every structural component of the kidney, including the glomerulus, tubules, interstitium, or renal microvasculature via distinct mechanisms, presenting with clinical manifestations ranging from asymptomatic elevation of serum biochemical markers (e.g., creatinine or cystatin C) and electrolyte imbalances to acute kidney injury (AKI) or chronic kidney disease necessitating renal replacement therapy [3]. Furthermore, the nephrotoxic potential of these agents may limit their therapeutic efficacy and increase patient morbidity and mortality. Thus, under-

Received: February 8, 2021; Revised: May 16, 2021; Accepted: May 17, 2021

Editor: Eun Hui Bae, Chonnam National University, Gwangju, Republic of Korea

Correspondence: Min Hee Hong

Division of Medical Oncology, Department of Internal Medicine, Yonsei Cancer Center, Severance Hospital, Yonsei University College of Medicine, 50-1 Yonsei-ro, Seodaemun-gu, Seoul 03722, Republic of Korea. E-mail:minhee_hong@yuhs.ac

ORCID: https://orcid.org/0000-0003-3490-2195

Copyright (C) 2021 by The Korean Society of Nephrology

(a) This is an Open Access article distributed under the terms of the Creative Commons Attribution Non-Commercial and No Derivatives License (http:// creativecommons.org/licenses/by-nc-nd/4.0/) which permits unrestricted non-commercial use, distribution of the material without any modifications, and reproduction in any medium, provided the original works properly cited. 
standing the nephrotoxicity of cancer therapeutics is critical for effectively managing oncologic patients.

The mechanisms underlying drug-induced nephrotoxicity associated with most cancer therapeutic drugs have not been thoroughly investigated or established. Hence, generating appropriate strategies to prevent or minimize nephrotoxic injuries, and to maintain the dose intensity of agents, can be challenging. In the present review, we collate the incidence, risk factors, clinical features, and management of drug-related nephrotoxicity of specific anticancer drugs based on the currently available literature, focusing on targeted molecular therapies and immunotherapies. With the growing usage of novel cancer therapies, oncologists and nephrologists should be aware of the attributes and characteristics of each chemotherapeutic drug. Table 1 summarizes the indications, mechanisms of action, and types of renal involvement of novel anticancer drugs.

\section{Targeted therapy-induced nephrotoxicity}

Molecular targeting anticancer therapies have been developed to exploit the oncogenic addictive nature of cancer and explicitly block the growth and spread of cancer by inhibiting specific molecules or pathways. Typically, these agents are associated with higher response rates than those by cytotoxic agents, with fewer adverse events. However, they have also been associated with various renal injuries.

\section{Epidermal growth factor receptor inhibitors}

Epidermal growth factor receptor (EGFR) is a transmembrane protein, and the binding of EGFR to its ligand results in phosphorylation of the EGFR tyrosine kinase domain, thus activating downstream pathways [4]. The most widely used agents that interfere with EGFR pathways are EGFR

Table 1. Novel cancer therapeutics and their renal manifestations

\begin{tabular}{|c|c|c|c|c|}
\hline Drug & $\begin{array}{l}\text { Target or mode of } \\
\text { action }\end{array}$ & U.S. FDA-approved indication & Renal manifestations & $\begin{array}{l}\text { Need for dosage } \\
\text { adjustment in renal } \\
\text { insufficiency }\end{array}$ \\
\hline $\begin{array}{l}\text { Gefitinib, erlotinib, afatinib, } \\
\text { osimertinib, dacomitinib }\end{array}$ & EGFR TKI & NSCLC & $\begin{array}{l}\text { Minimal change disease, mem- } \\
\text { branous nephropathy (rare) }\end{array}$ & No \\
\hline Cetuximab, panitumumab & EGFR mAb & $\begin{array}{l}\text { Head and neck cancer, col- } \\
\text { orectal cancer }\end{array}$ & Hypomagnesemia & No \\
\hline $\begin{array}{l}\text { Crizotinib, ceritinib, brigatinib, } \\
\text { alectinib, lorlatinib }\end{array}$ & ALK TKI & NSCLC & $\begin{array}{l}\text { Renal cyst (crizotinib), pseudo } \\
\text { or true AKI, Acute tubular } \\
\text { necrosis }\end{array}$ & Yes in severe cases \\
\hline Imatinib & $\begin{array}{l}\text { BCR-ABL and PDGFR } \\
\text { TKI }\end{array}$ & $\begin{array}{l}\text { Chronic myelogenous leuke- } \\
\text { mia, gastrointestinal stromal } \\
\text { tumor }\end{array}$ & AKI & Yes \\
\hline Dasatinib & $\begin{array}{l}\text { BCR-ABL and PDGFR, } \\
\text { VEGF TKI }\end{array}$ & Chronic myelogenous leukemia & AKI, proteinuria, TMA & No \\
\hline $\begin{array}{l}\text { Bevacizumab, ramucirumab, } \\
\text { aflibercept }\end{array}$ & $\begin{array}{l}\text { VEGF ligand binding } \\
\text { inhibition }\end{array}$ & Colorectal cancer, NSCLC & $\begin{array}{l}\text { Proteinuria, TMA, hypertension, } \\
\text { AKI, glomerulonephropathy }\end{array}$ & No \\
\hline $\begin{array}{l}\text { Sunitinib, sorafenib, axitinib, } \\
\text { pazopanib }\end{array}$ & VEGF receptor TKI & RCC, hepatocellular carcinoma & $\begin{array}{r}\text { Proteinuria, hypertension, AKI, } \\
\text { glomerulonephropathy, TMA }\end{array}$ & No \\
\hline Everolimus, temsirolimus & mTOR inhibitor & $\begin{array}{l}\text { RCC, breast cancer, neuroen- } \\
\text { docrine tumor }\end{array}$ & $\begin{array}{l}\text { Proteinuria, hypertension, AKI, } \\
\text { glomerulonephropathy, acute } \\
\text { tubular necrosis }\end{array}$ & No \\
\hline Dabrafenib, vemurafenib & BRAF inhibitor & Melanoma & AKI, hypokalemia, hyponatremia & No \\
\hline Pembrolizumab, nivolumab & PD-1 inhibitor & $\begin{array}{l}\text { NSCLC, SCLC, melanoma, RCC, } \\
\text { bladder cancer, and so on }\end{array}$ & $\begin{array}{l}\text { AKI, glomerulonephropathy } \\
\text { acute interstitial nephritis }\end{array}$ & No \\
\hline $\begin{array}{l}\text { Atezolizumab, durvalumab, } \\
\text { avelumab }\end{array}$ & PD-L1 inhibitor & $\begin{array}{l}\text { NSCLC, SCLC, RCC, bladder } \\
\text { cancer, and so on }\end{array}$ & $\begin{array}{l}\text { AKI, glomerulonephropathy } \\
\text { acute interstitial nephritis }\end{array}$ & No \\
\hline Ipilimumab & CTLA-4 inhibitor & Melanoma, NSCLC, RCC & $\begin{array}{l}\text { AKI, glomerulonephropathy } \\
\text { acute interstitial nephritis }\end{array}$ & No \\
\hline
\end{tabular}

AKI, acute kidney injury; ALK, anaplastic lymphoma kinase; BCR-ABL, breakpoint cluster region-Abelson leukemia; BRAF, v-Raf murine sarcoma viral oncogene homolog B; CTLA-4, cytotoxic T-lymphocyte antigen-4; EGFR, epidermal growth factor receptor; mAb, monoclonal antibody; mTOR, mammalian target of rapamycin; NSCLC, non-small cell lung cancer; PD-1, programmed cell death protein-1; PD-L1, programmed death-ligand 1; PDGFR, platelet-derived growth factor receptor; RCC, renal cell carcinoma; SCLC, small cell lung cancer; TKI, tyrosine kinase inhibitor; TMA, thrombotic microangiopathy; U.S. FDA, U.S. Food and Drug Administration; VEGF, vascular endothelial growth factor. 
tyrosine kinase inhibitors (EGFR-TKIs) targeting EGFR mutations in non-small cell lung cancer (NSCLC), including gefitinib, erlotinib, afatinib, dacomitinib, osimertinib, and the recently developed lazertinib [5]. EGFR mutations reportedly account for more than $40 \%$ of Asian NSCLC cases and approximately $20 \%$ of NSCLC cases in Western countries [6]. EGFR-TKIs predominantly undergo hepatic metabolism, with less than $4 \%$ undergoing renal excretion; thus, these agents can be safely used in patients with decreased renal function without necessitating dose adjustment $[7,8]$. In some case reports, these drug families have been safely used in patients with end-stage renal disease receiving hemodialysis $[9,10]$. Despite their excellent safety profile, rare glomerular injuries such as minimal change disease and membranous nephropathy associated with gefitinib have been reported in a case report series [11,12]. Although these events were mainly reported in Asian patients, whether ethnic differences or specific EGFR-TKIs can be linked to these events remains unknown.

EGFR monoclonal antibodies targeting EGFR, such as cetuximab, panitumumab, or necitumumab, are indicated in patients with colon cancer, NSCLC, and head and neck squamous cell carcinoma; typically, these agents are not associated with a decline in renal function [13]. However, EGFR monoclonal antibodies are well known for their capacity to induce magnesium wasting as these agents prevent EGFR activation in the distal tubule, thus resulting in renal magnesium wasting [14]. One meta-analysis study ( $\mathrm{n}=$ 3,081 ) reported that the overall incidence of hypomagnesemia in patients treated with cetuximab was $36 \%$, presenting grade 3 or more hypomagnesemia (5.6\%) [15]. Furthermore, the frequency of hypomagnesemia was reportedly higher with panitumumab therapy and was associated with other adverse events such as diarrhea or dehydration. However, hypomagnesemia was not related to serious irreversible complications [16].

\section{Anaplastic lymphoma kinase inhibitors}

Anaplastic lymphoma kinase (ALK) fusions have been observed in various malignancies, including NSCLC, Hodgkin lymphoma, anaplastic large-cell lymphoma, and sarcoma. ALK fusions have been observed in $4 \%$ to $6 \%$ of NSCLC cases, and the advent of ALK inhibitors has profoundly altered the management of ALK-positive NSCLC. Currently, ALK inhibitors approved by the U.S. Food and Drug Administration (FDA) include crizotinib, ceritinib, alectinib, brigatinib, and lorlatinib, and these agents are known to be associated with renal cysts, pseudo or true AKI, peripheral edema, and hypophosphatemia.

Although the mechanism implicated in the growth of renal cysts remains to be clarified, crizotinib has been shown to result in renal cysts and to induce renal cyst progression. According to a safety database of 1,375 patients, $9 \%$ of patients treated with crizotinib acquired new cysts within 6 months of treatment initiation [17]. Furthermore, 2\% of patients with preexisting cysts developed new cysts or experienced enlargement of preexisting cysts. Additionally, the risk of renal cyst formation or progression was increased in Korean patients [17]. As these phenomena have not been observed with other ALK inhibitors, including ceritinib, brigatinib, and lorlatinib, they have been considered specific to crizotinib. However, a recent case described alectinib usage and renal cyst development [18]. As these lesions can be easily mistaken for lung cancer progression or development of primary renal cell carcinoma (RCC), clinical awareness is essential to avoid cessation of an otherwise efficacious drug. Generally, close monitoring of renal cyst development is recommended without dose adjustment or interruption, and most patients can continue ALK inhibitor treatment.

Another interesting observation related to ALK inhibitors is the presence of pseudo or true AKI. A large retrospective analysis of 1,868 patients reported an acute decline in renal function within 2 weeks of crizotinib initiation, with minimal cumulative effects and was mostly reversible after treatment discontinuation [19]. Camidge et al. [20] suggested that the decline in creatinine clearance reflects creatinine excretion rather than true renal injury, thus termed pseudo AKI. An in vitro study demonstrated that crizotinib impeded renal creatinine excretion by the organic cation transporter 2 in the proximal tubule [21]. Some patients demonstrate normal glomerular filtration rates when non-creatinine-based measurements (iothalamate assessment) of glomerular filtration rate are performed [20]. Therefore, most patients with elevated serum creatinine levels can continue crizotinib without dose adjustment under close monitoring. However, ALK-TKIs can also induce true kidney injury associated with acute tubular necrosis; thus, the use of a non-creatinine-based assessment of renal function should be considered before the final decision [22,23]. 
Breakpoint cluster region-Abelson leukemia and KIT tyrosine kinase inhibitors

Imatinib mesylate was the first approved TKI that targets the gene product of breakpoint cluster region-Abelson leukemia (BCR-ABL) in chronic myeloid leukemia, with additional activity on KIT and platelet-derived growth factor receptor (PDGFR). Several cases of imatinib-related nephrotoxicity have been reported [24]. Two possible mechanisms have been proposed; tumor lysis syndrome and toxic tubular damage, and tubular damage possibly associated with PDGFR inhibition by imatinib. Among 105 patients treated with imatinib and a median follow-up of 4.5 years, $7 \%$ of patients reportedly experienced AKI, with $12 \%$ of patients presenting with chronic kidney disease [25]. Treatment duration may be a determinant of the declining glomerular filtration rate. Oncologists and nephrologists should be aware that long-term treatment with imatinib may cause an irreversible and clinically significant decrease in the glomerular filtration rate, as well as chronic kidney disease. Close attention should be paid to avoid the concomitant use of nephrotoxic agents and dehydrating conditions, and clinicians in charge of these patients should regularly monitor renal function.

Dasatinib, a second-generation TKI indicated for chronic myelogenous leukemia, is known to inhibit vascular endothelial growth factor (VEGF), along with BCR-ABL and PDGFR. In a phase I trial $(\mathrm{n}=33), 12 \%$ of patients developed proteinuria, with $3 \%$ presenting grades 3 and 4; the proposed underlying mechanisms include VEGF co-inhibition or podocyte disruption [26-28]. Ochiai et al. [29] reported that dasatinib induced nephrotic syndrome, with complete recovery observed after switching to nilotinib. Switching to imatinib or nilotinib, which are believed to be unrelated to proteinuria development, is currently a popular management strategy for dasatinib-associated proteinuria.

\section{Vascular endothelial growth factor inhibitors}

VEGF A and VEGF receptors (VEGFR) play a major role in angiogenesis, promoting cancer cell proliferation, migration, and invasion. There are two kinds of VEGF pathway inhibitors: VEGF ligand inhibitors, such as bevacizumab, ramucirumab, and aflibercept; and tyrosine kinase inhibitors that block the VEGFR intracellular kinase domain, including sunitinib, sorafenib, and axitinib. Various renal effects have been observed following treatment with VEGF pathway inhibitors.

Notably, the occurrence of proteinuria after inhibiting VEGF signaling pathways represents the importance of the VEGF pathway in normal kidney functions. Both monoclonal antibodies and TKIs are known to induce proteinuria, with frequency and severity differing slightly between drugs. Among these agents, bevacizumab has been extensively investigated, and frequencies of all proteinuria grades were $21 \%$ to $62 \%$, with individuals presenting with grade 3 or more proteinuria comprising $2 \%$ to $6.5 \%$ [30-32]. The severity of proteinuria appears to be dose-dependent and generally reversible. However, persistent proteinuria is not unusual. Thus, in the case of nephrotic syndrome, bevacizumab must be discontinued permanently $[30,33,34]$. Underlying diseases, such as chronic kidney disease and RCC, may increase the risk of proteinuria. Furthermore, the combination of cytotoxic chemotherapy may worsen the degree of proteinuria. Regular monitoring via urinary analysis of proteinuria prior to each cycle is recommended [34]. Table 2 summarizes the management of proteinuria. Common glomerulopathies associated with VEGF inhibition include intraglomerular thrombotic microangiopathy, minimal change disease, and focal segmental glomerulosclerosis (FSGS) [35]. The use of angiotensin receptor blockers or angiotensin-converting enzyme inhibitors may be recommended for renoprotection. However, these agents have not been investigated in randomized placebo-controlled prospective trials for the management of VEGF inhibitor-associated proteinuria or glomerulonephropathy [34].

Hypertension has been observed in $8 \%$ to $36 \%$ of patients, with grade 3 or 4 hypertension ranging from $1.8 \%$ to $22 \%$ [32,36,37]. Increased risk of hypertension might be dose-dependent and associated with cancer types such as RCC, NSCLC, and pancreatic cancer [37]. Recent data suggest that patients who develop hypertension during VEGF inhibition might experience more effective VEGF antagonism, resulting in superior anticancer activity. The development of hypertension is associated with better tumor control and survival [38-40]. The management of hypertension is summarized in Table 2, and blood pressure should be monitored and managed when hypertension is detected [34]. Except for patients in hypertensive crisis, clinicians should attempt continuing VEGF inhibitor treatment without interruption by administering suitable antihypertensive drugs. There are 
Table 2. Guidelines for the management of proteinuria and hypertension in patients receiving VEGF inhibitors

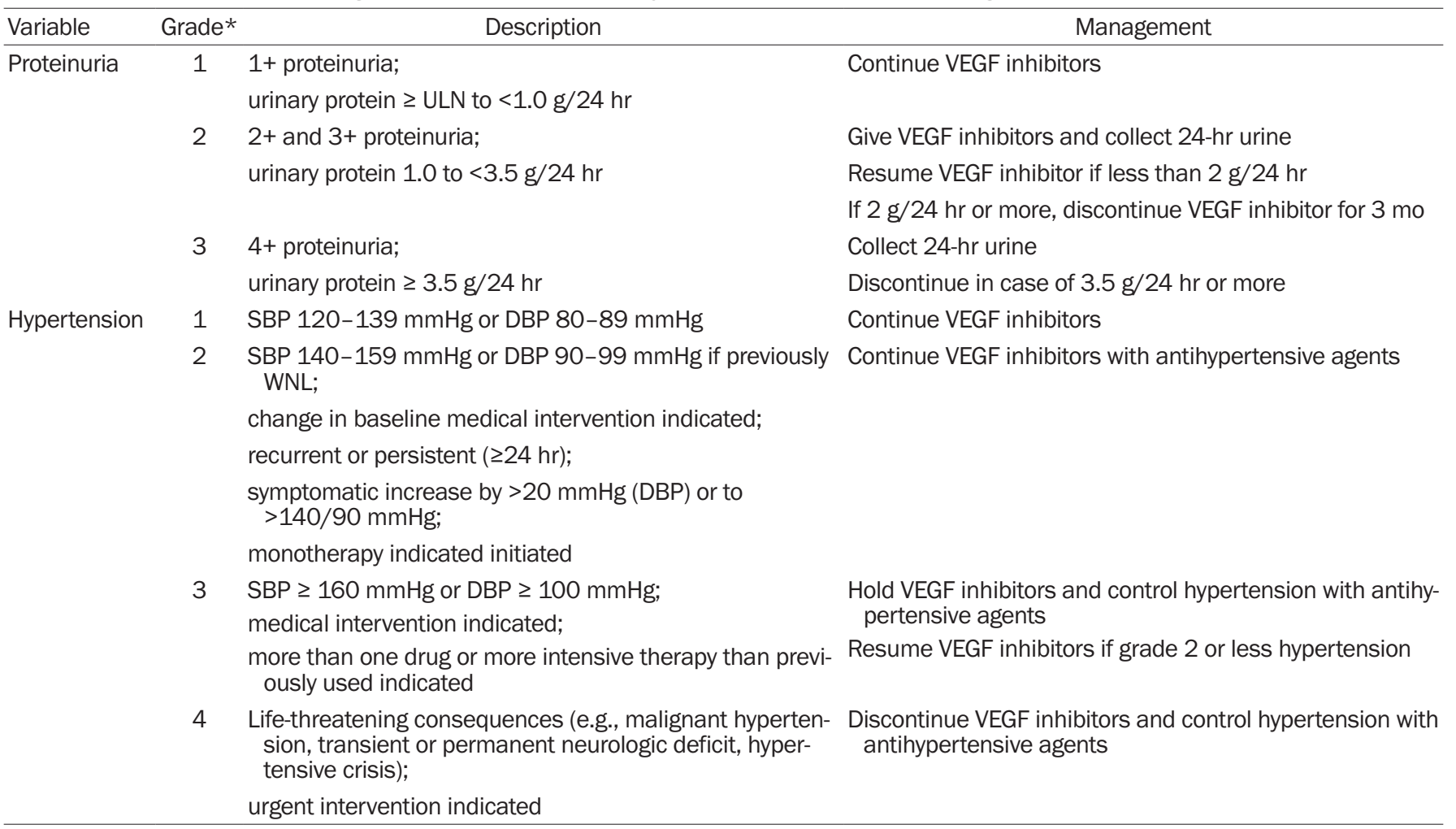

*Grading follows the National Cancer Institute-Common Toxicity Criteria (NCl-CTC) version 5.0.

DBP, diastolic blood pressure; SBP, systolic blood pressure; ULN, upper limit of normal; VEGF, vascular endothelial growth factor; WNL, within normal limits.

no evidence-based guidelines for selecting the most appropriate antihypertensive agents to manage and treat VEGF inhibitor-induced hypertension [41].

\section{Mammalian target of rapamycin inhibitors}

A serine/threonine protein kinase, termed mammalian target of rapamycin (mTOR), is implicated in RCC [42]. mTOR inhibitors, everolimus and temsirolimus, are known to be associated with proteinuria, hypertension, and renal failure, and their proposed mechanisms include VEGF pathway disruption [43]. Large prospective everolimus studies reported proteinuria ranging from $10 \%$ to $14 \%$ and grade 3 or more of proteinuria occurred approximately $1 \%$ or $2 \%[44,45]$. However, there have been no reports of nephrotic syndrome. These studies also reported that the frequencies of all grades of hypertension were $8 \%$ to $10 \%$ and that patients with hypertension grade 3 or more comprised $1 \%$ to $4 \%$. In the case of temsirolimus, the incidence of proteinuria has not been formally reported, but Izzedine et al. [46] reported four pa- tients with acute kidney injuries, along with biopsy-proven acute tubular necrosis during mTOR inhibitor treatment with everolimus and temsirolimus. Moreover, FSGS was observed in a patient receiving temsirolimus [47]. Worsening AKI was reported in $57 \%$ of patients (with grade 3 to 4 AKI in approximately $3 \%$ of patients) in temsirolimus treatment [48]. As patients with RCC may be elderly and many have undergone previous nephrectomy, such patients are at high risk of developing renal failure. Thus, although the incidence is low and most cases are mild, regular monitoring of high blood pressure, proteinuria, and worsening renal function are recommended for mTOR inhibitor treatment, especially in patients with RCC.

\section{The BRAF inhibitor}

The v-Raf murine sarcoma viral oncogene homolog B (BRAF), one of three members of the RAF kinase family, plays an essential role in the carcinogenesis pathway [49]. The BRAF ${ }^{\mathrm{V} 600 \mathrm{E}}$ mutation, which substitutes glutamic acid for 
valine at codon 600 , accounts for $40 \%$ to $60 \%$ of melanoma and $1 \%$ of NSCLC cases $[50,51]$. Dabrafenib and vemurafenib are standard treatments for metastatic $\mathrm{BRAF}^{\mathrm{V} 600 \mathrm{E}}$-mutant melanoma [52]. Although renal insufficiency has not been reported in large prospective trials and the true incidence might be very low, AKI related to vemurafenib has been noted in several case series [53-55]. The Food and Drug Administration Adverse Event Reporting System (FAERS) database, maintained by the U.S. FDA, has recorded 132 and 13 AKI cases following treatment with vemurafenib and dabrafenib, respectively, between 2011 and 2014 [56]. Vemurafenib seems to induce greater nephrotoxicity than dabrafenib. Hypokalemia and hyponatremia were also observed at an extremely low frequency. Based on available data, renal function should be monitored during BRAF inhibitor treatment.

\section{Nephrotoxicity of immunotherapies}

The expanding use of immune checkpoint inhibitors (ICIs), including programmed cell death protein-1 (PD-1)/programmed death-ligand 1 (PD-L1) inhibitors and cytotoxic T-lymphocyte antigen-4 (CTLA-4) inhibitors, has altered the treatment paradigms for several cancer types [57]. Compared with conventional cytotoxic chemotherapy, immunotherapy has a favorable safety profile, with most patients experiencing only mild adverse events. However, unusual phenomena have been observed-so-called immune-related adverse events (irAEs) -including immune-mediated pneumonitis, hypothyroidism, hyperthyroidism, infusion reaction, colitis, myositis, and rashes [58]. Generally, the incidence and severity of irAEs are higher with CTLA-4 inhibitor monotherapy and ICI combination than those with PD-1/PD-L1 blockade monotherapy [59]. This autoimmune activity also involves the kidneys, but at a lower incidence than other organs, such as the thyroid, lung, or colon. Renal irAEs include AKI, proteinuria, and electrolyte imbalance [60]. In a meta-analysis of 11,482 patients in $48 \mathrm{PD}-1$ inhibitor clinical trials, the overall AKI incidence was $2.2 \%$, with hypocalcemia observed in $1.0 \%$ [61]. In patients with AKI treated with PD-1 inhibitors, the estimated rate of interstitial nephritis was $16.6 \%$. In one series that included 574 melanoma patients treated with nivolumab, the overall incidence of nephrotoxicity was $2 \%$, with a median onset time of 15.1 weeks after treatment initiation [62]. In another series of 3,695 patients treated with various ICIs, the overall incidence of AKI was $2.2 \%$, and it was more frequent in patients receiving combination therapy with ipilimumab and nivolumab (4.9\%) than that in patients receiving ipilimumab (2.0\%), nivolumab (1.9\%), and pembrolizumab (1.4\%) [63]. Wanchoo et al. [64] reported that acute interstitial nephritis (AIN) occurs 6 to 12 weeks and 3 to 12 months after initiation of CTLA-4 and PD-1 inhibitors, respectively. These data suggest that the incidence of nephrotoxicity in ICI treatment is generally low, but higher incidence can be observed following combination therapy. Interestingly, ICI-induced AKI sometimes follows other extrarenal irAEs, such as rash, thyroiditis, and colitis $[65,66]$. The concomitant use of proton pump inhibitors and lower baseline glomerular filtration rate have been indicated as potential risk factors [63,65-67]. According to the current literature, the most common kidney lesion is AIN, followed by minimal change disease, thrombotic microangiopathy, lupus-like nephritis, and FSGS [63,67-70]. ICI-induced AIN is characterized by its variable onset and frequent relapse when compared with traditional drug-induced AIN, highlighting a unique mechanism of action for ICIs [63]. Additionally, ICI-induced AIN is characterized by its excellent response to steroids. However, its frequent relapsing nature requires long-term corticosteroid therapy [71].

The precise mechanisms of immune-related nephrotoxicity remain unknown. However, self-antigen-specific T cell activation involving various components of the kidney is one suggested mechanism [64]. Data regarding the management of ICI-induced nephritis are limited, but glucocorticoids are the mainstay of treatment. ICI therapy should be withheld following grade 2 or more nephritis. Although the optimal dose and duration remain unclear, prednisone 0.5 to $2 \mathrm{mg} /$ $\mathrm{kg} /$ day is recommended in guidelines $[72,73]$. Table 3 summarizes the management of ICI-induced nephrotoxicity [72].

An important issue in clinical practice is administering ICIs to patients with solid organ transplants, especially renal allografts. In several prospective clinical trials involving ICI, patients with organ transplantation have been excluded, and their safety in organ transplant recipients is not well defined and established $[57,58]$. However, a high incidence of renal allograft rejection (40.9\%) was reported in 44 kidney transplant patients administered ICIs, with a higher rate recorded in those receiving PD-1/PD-L1 inhibitors (40.7\%) than in those administering CTLA-4 inhibitors (22.2\%). Among these 44 patients, 15 (34.1\%) experienced allograft failure and 8 (18.1\%) died [74]. Based on the observed implications of PD-1/PD-L1 signaling in solid organ transplant, 
Table 3. Management of renal immune-related adverse events of immune checkpoint inhibitor

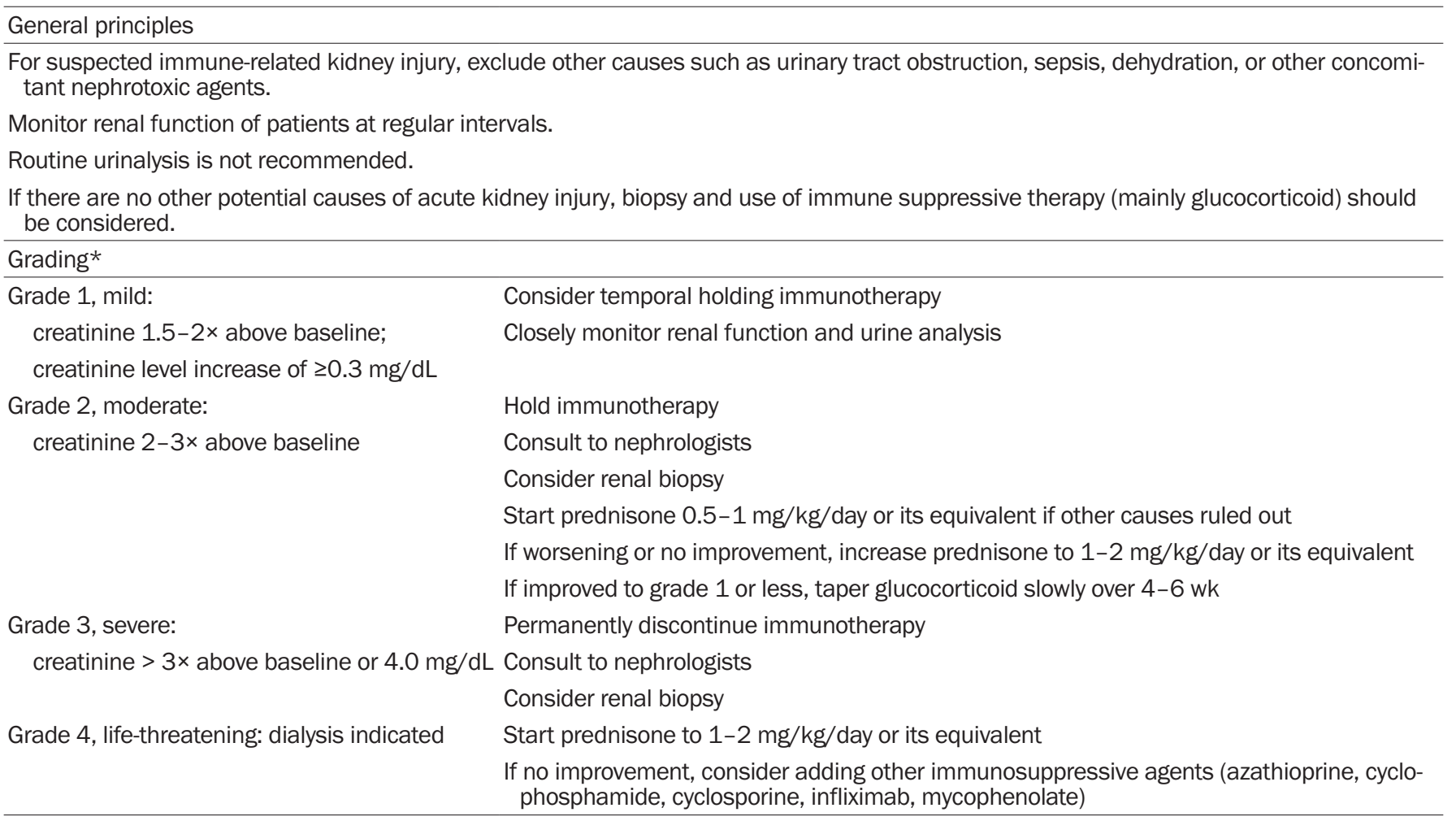

* Grading follows the National Comprehensive Cancer Network. Management of Immunotherapy-Related Toxicities (version 1.2021). Available at: https:// www.nccn.org/professionals/physician_gls/pdf/immunotherapy.pdf (accessed 2021 Feb 1).

blockade of the PD-1/PD-L1 pathway may result in a higher rate of graft rejection than CTLA-4 blockade [75]. Another case series of 23 patients reported a similar renal allograft rejection rate of $47 \%$, with $81 \%$ graft loss and $46 \%$ death [76]. The median time from ICI initiation to acute rejection was less than a month in both reports $[74,76]$. At present, the exact mechanism of graft rejection remains to be elucidated. Graft rejection is a complex process involving both humoral immunity and cellular immunity, and both the CTLA- 4 and PD-1 pathways are implicated in the immune tolerance of transplanted organ [77]. Since their positions in the immune tolerant context differ, the proposed mechanisms are considered distinct between PD-1 inhibitors and CTLA- 4 inhibitors [60]. PD-L1 is known to be essential for peripheral graft tolerance and protection from chronic rejection and its pathway inhibition produces the activation of cellular immunity via the effector $\mathrm{T}$ cell and the downregulation of regulatory $\mathrm{T}$ cell, subsequently inducing allograft rejection. On the other hand, the suggested mechanism of CTLA- 4 inhibition is that CTLA-4 inhibitor could prime and generate new donor-specific $\mathrm{T}$ cell activation and cytotoxicity, and guard alloreactive
T cells against apoptotic death, ultimately prompting rejection [78]. Despite a paucity of data, prior history of rejection, presence of donor-specific antibodies, and PD-Ll expression in transplant patients have been associated with increased risk of rejection $[79,80]$. High allograft rejection shortly after ICI initiation accompanied by a high mortality rate has led to the development of preventive approaches, including close monitoring of renal function and preemptive immunosuppressant usage in joint with ICI treatment. However, there are no current management guidelines regarding this issue. Some authors recommend careful monitoring of kidney function, such as frequent serum creatinine measurement starting on a weekly basis and then switching to biweekly or monthly if renal function remains stable [60]. In the presence of worsening renal function without other causes, renal biopsy should be considered, and careful administration of contrast dye with appropriate hydration during computed tomography imaging evaluation is suggested. The treatment of ICI in transplant recipients should be thoroughly discussed with patients about the potential benefit and risk of graft loss before initiation. 
Other types of immunotherapies include chimeric antigen receptor T (CAR-T) cell therapy and cytokine therapy. Exogenous cytokines gained popularity in the 1980s, and only two cytokines, interferon-alpha (IFN- $\alpha$ ) and high-dose interleukin-2 (IL-2), received the U.S. FDA approval for indications such as malignant melanoma, RCC, and chronic myelogenous leukemia [81]. IFN- $\alpha$ triggers T cell-mediated responses and results in renal inflammation, and is thus complicated by several types of kidney injury [82]. Minimal change disease, FSGS, and thrombotic microangiopathy are associated with IFN- $\alpha$ treatment and have been reported in select cases [71]. Prompt recognition and treatment discontinuation remain critical measures [83]. Another approved cytokine, high-dose IL-2, causes moderate to severe hypotension, with a frequency of $60 \%$ to $90 \%$. Capillary leak syndrome can also occur, which can induce intravascular volume depletion, hypotension, and kidney injury following IL-2 therapy [84]. Thus, capillary leakage can bring about prerenal AKI, and severe hypotension can contribute to acute tubular injury [71]. Appropriate fluid therapy should be employed with IL-2 therapy [85]. However, the absence of biomarkers, low overall response rates, and high frequency of severe adverse events have replaced the use of cytokine anticancer treatment with ICIs [60].

The incidence of nephrotoxicity of another novel immunotherapeutic, CAR-T cell therapy, has been less well established. In one series of 46 patients with non-Hodgkin lymphoma, the incidence of AKI was 30\% [86]. Another report revealed that AKI incidence in 78 patients with diffuse large B-cell lymphoma was 19\% (15 patients), including six acute tubular necrosis events. Hypophosphatemia, hypokalemia, and hyponatremia reportedly occurred in more than $50 \%$ of patients [87]. Currently, no direct glomerular or tubular injuries have been reported [86]. However, systemic toxicities, including tumor lysis syndrome and cytokine release syndrome associated with hypotension, liver dysfunction, cardiac dysfunction, and intravascular volume depletion can cause AKI and electrolyte imbalances [71]. Early recognition and appropriate fluid therapy are crucial for managing CAR-T therapy-associated kidney injury [86].

\section{Summary}

Chemotherapy-induced kidney injury is a common problem encountered by medical oncologists and nephrologists. The advent of novel anticancer drugs such as targeted molecular agents and immune checkpoint modulators has improved patient survival while simultaneously increasing the prevalence of renal injuries. Despite their relatively low incidence, it is crucial for medical oncologists and nephrologists to be familiar with possible renal toxicities and complications and regularly monitor renal function in patients exposed to these agents.

\section{Conflicts of interest}

The author has no conflicts of interest to declare.

\section{ORCID}

Min Hee Hong, https://orcid.org/0000-0003-3490-2195

\section{References}

1. Soria JC, Ohe Y, Vansteenkiste J, et al. Osimertinib in untreated EGFR-mutated advanced non-small-cell lung cancer. $N$ Engl J Med 2018;378:113-125.

2. Reck M, Rodríguez-Abreu D, Robinson AG, et al. Pembrolizumab versus chemotherapy for PD-L1-positive non-small-cell lung cancer. N Engl J Med 2016;375:1823-1833.

3. Barnett LMA, Cummings BS. Nephrotoxicity and renal pathophysiology: a contemporary perspective. Toxicol Sci 2018;164:379-390.

4. Normanno N, De Luca A, Bianco C, et al. Epidermal growth factor receptor (EGFR) signaling in cancer. Gene 2006;366:2-16.

5. Ahn MJ, Han JY, Lee KH, et al. Lazertinib in patients with EGFR mutation-positive advanced non-small-cell lung cancer: results from the dose escalation and dose expansion parts of a first-inhuman, open-label, multicentre, phase 1-2 study. Lancet Oncol 2019;20:1681-1690.

6. Mitsudomi T. Molecular epidemiology of lung cancer and geographic variations with special reference to EGFR mutations. Transl Lung Cancer Res 2014;3:205-211.

7. Yoshimura M, Nakamura S, Imamura F, Ueno K, Yamamoto S, Igarashi T. Severe myelotoxicity in a combination of gefitinib and vinorelbine. Lung Cancer 2004;45:121-123.

8. Miller AA, Murry DJ, Owzar K, et al. Phase I and pharmacokinetic study of erlotinib for solid tumors in patients with hepatic or renal dysfunction: CALGB 60101. J Clin Oncol 2007;25:30553060. 
9. Imai H, Kaira K, Naruse I, et al. Successful afatinib treatment of advanced non-small-cell lung cancer patients undergoing hemodialysis. Cancer Chemother Pharmacol 2017;79:209-213.

10. Bersanelli M, Tiseo M, Artioli F, Lucchi L, Ardizzoni A. Gefitinib and afatinib treatment in an advanced non-small cell lung cancer (NSCLC) patient undergoing hemodialysis. Anticancer Res 2014;34:3185-3188.

11. Kaneko T, Shimizu A, Aoki M, Tsuruoka S. A case of gefitinib-associated membranous nephropathy in treatment for pulmonary adenocarcinoma. CEN Case Rep 2015;4:31-37.

12. Maruyama K, Chinda J, Kuroshima T, et al. Minimal change nephrotic syndrome associated with gefitinib and a successful switch to erlotinib. Intern Med 2015;54:823-826.

13. Ciardiello F, Tortora G. EGFR antagonists in cancer treatment. $N$ Engl J Med 2008;358:1160-1174.

14. Groenestege WM, Thébault S, van der Wijst J, et al. Impaired basolateral sorting of pro-EGF causes isolated recessive renal hypomagnesemia. J Clin Invest 2007;117:2260-2267.

15. Cao Y, Liao C, Tan A, Liu L, Gao F. Meta-analysis of incidence and risk of hypomagnesemia with cetuximab for advanced cancer. Chemotherapy 2010;56:459-465.

16. Petrelli F, Borgonovo K, Cabiddu M, Ghilardi M, Barni S. Risk of anti-EGFR monoclonal antibody-related hypomagnesemia: systematic review and pooled analysis of randomized studies. Expert Opin Drug Saf2012;11 Suppl 1:S9-S19.

17. Schnell P, Bartlett CH, Solomon BJ, et al. Complex renal cysts associated with crizotinib treatment. Cancer Med 2015;4:887-896.

18. Longo V, Catino AM, Montrone M, et al. Development of complex renal cysts during crizotinib treatment and also during alectinib treatment: a possible drug class effect? J Thorac Oncol 2019;14:e170.

19. Camidge DR, Kim EE, Usari T, Polli A, Lewis I, Wilner KD. Renal effects of crizotinib in patients with ALK-positive advanced NSCLC. J Thorac Oncol 2019;14:1077-1085.

20. Camidge DR, Brosnan EM, DeSilva C, Koo PJ, Chonchol M. Crizotinib effects on creatinine and non-creatinine-based measures of glomerular filtration rate. J Thorac Oncol 2014;9:16341637.

21. Arakawa H, Omote S, Tamai I. Inhibitory effect of crizotinib on creatinine uptake by renal secretory transporter OCT2.J Pharm Sci 2017;106:2899-2903.

22. Gastaud L, Ambrosetti D, Otto J, et al. Acute kidney injury following crizotinib administration for non-small-cell lung carcinoma. Lung Cancer 2013;82:362-364.

23. Ramachandran P, Morcus R, Tahir M, Onukogu I, Spinowitz
B, Wang JC. Alectinib (Alecensa)-induced reversible grade IV nephrotoxicity: a case report and review of the literature. $J$ Med Case Rep 2018;12:303.

24. Abbas A, Mirza MM, Ganti AK, Tendulkar K. Renal toxicities of targeted therapies. Target Oncol 2015;10:487-499.

25. Marcolino MS, Boersma E, Clementino NC, et al. Imatinib treatment duration is related to decreased estimated glomerular filtration rate in chronic myeloid leukemia patients. Ann Oncol 2011;22:2073-2079.

26. Demetri GD, Lo Russo P, MacPherson IR, et al. Phase I dose-escalation and pharmacokinetic study of dasatinib in patients with advanced solid tumors. Clin Cancer Res 2009;15:6232-6240.

27. Ozkurt S, Temiz G, Acikalin MF, Soydan M. Acute renal failure under dasatinib therapy. Ren Fail 2010;32:147-149.

28. Calizo RC, Bhattacharya S, van Hasselt JGC, et al. Disruption of podocyte cytoskeletal biomechanics by dasatinib leads to nephrotoxicity. Nat Commun 2019;10:2061.

29. Ochiai S, Sato Y, Minakawa A, Fukuda A, Fujimoto S. Dasatinib-induced nephrotic syndrome in a patient with chronic myelogenous leukemia: a case report. BMC Nephrol 2019;20:87.

30. Wu S, Kim C, Baer L, Zhu X. Bevacizumab increases risk for severe proteinuria in cancer patients. J Am Soc Nephrol 2010; 21:1381-1389.

31. Izzedine H, Massard C, Spano JP, Goldwasser F, Khayat D, Soria JC. VEGF signalling inhibition-induced proteinuria: mechanisms, significance and management. Eur J Cancer 2010;46:439448.

32. Zhao T, Wang X, Xu T, Xu X, Liu Z. Bevacizumab significantly increases the risks of hypertension and proteinuria in cancer patients: a systematic review and comprehensive meta-analysis. Oncotarget 2017;8:51492-51506.

33. Saif MW, Mehra R. Incidence and management of bevacizumab-related toxicities in colorectal cancer. Expert Opin Drug Saf 2006;5:553-566.

34. Miles D, Bridgewater J, Ellis P, et al. Using bevacizumab to treat metastatic cancer: UK consensus guidelines. Br J Hosp Med (Lond) 2010;71:670-677.

35. Izzedine H, Escudier B, Lhomme C, et al. Kidney diseases associated with anti-vascular endothelial growth factor (VEGF): an 8-year observational study at a single center. Medicine (Baltimore) 2014;93:333-339.

36. Li M, Kroetz DL. Bevacizumab-induced hypertension: clinical presentation and molecular understanding. Pharmacol Ther 2018;182:152-160.

37. Ranpura V, Pulipati B, Chu D, Zhu X, Wu S. Increased risk of 
high-grade hypertension with bevacizumab in cancer patients: a meta-analysis. Am J Hypertens 2010;23:460-468.

38. Miyake M, Kuwada M, Hori S, et al. The best objective response of target lesions and the incidence of treatment-related hypertension are associated with the survival of patients with metastatic renal cell carcinoma treated with sunitinib: a Japanese retrospective study. BMC Res Notes 2016;9:79.

39. Rini BI, Schiller JH, Fruehauf JP, et al. Diastolic blood pressure as a biomarker of axitinib efficacy in solid tumors. Clin Cancer Res 2011;17:3841-3849.

40. Dionísio de Sousa IJ, Ferreira J, Rodrigues J, et al. Association between bevacizumab-related hypertension and response to treatment in patients with metastatic colorectal cancer. ESMO Open 2016;1:e000045.

41. Syrigos KN, Karapanagiotou E, Boura P, Manegold C, Harrington K. Bevacizumab-induced hypertension: pathogenesis and management. BioDrugs 2011;25:159-169.

42. Motzer RJ, Escudier B, Oudard S, et al. Efficacy of everolimus in advanced renal cell carcinoma: a double-blind, randomised, placebo-controlled phase III trial. Lancet 2008;372:449-456.

43. Letavernier E, Legendre C. mToR inhibitors-induced proteinuria: mechanisms, significance, and management. Transplant Rev (Orlando) 2008;22:125-130.

44. Choueiri TK, Escudier B, Powles T, et al. Cabozantinib versus everolimus in advanced renal cell carcinoma (METEOR): final results from a randomised, open-label, phase 3 trial. Lancet $\mathrm{On}$ col 2016;17:917-927.

45. Motzer RJ, Hutson TE, Glen H, et al. Lenvatinib, everolimus, and the combination in patients with metastatic renal cell carcinoma: a randomised, phase 2, open-label, multicentre trial. Lancet Oncol 2015;16:1473-1482.

46. Izzedine H, Escudier B, Rouvier P, et al. Acute tubular necrosis associated with mTOR inhibitor therapy: a real entity biopsy-proven. Ann Oncol 2013;24:2421-2425.

47. Izzedine H, Boostandoot E, Spano JP, Bardier A, Khayat D. Temsirolimus-induced glomerulopathy. Oncology 2009;76:170-172.

48. Kwitkowski VE, Prowell TM, Ibrahim A, et al. FDA approval summary: temsirolimus as treatment for advanced renal cell carcinoma. Oncologist 2010;15:428-435.

49. Cantwell-Dorris ER, O'Leary JJ, Sheils OM. BRAFV600E: implications for carcinogenesis and molecular therapy. Mol Cancer Ther 2011;10:385-394.

50. Ascierto PA, Kirkwood JM, Grob JJ, et al. The role of BRAF V600 mutation in melanoma. J Transl Med 2012;10:85.

51. Planchard D, Smit EF, Groen HJM, et al. Dabrafenib plus trame- tinib in patients with previously untreated BRAFV600E-mutant metastatic non-small-cell lung cancer: an open-label, phase 2 trial. Lancet Oncol 2017;18:1307-1316.

52. Seth R, Messersmith H, Kaur V, et al. Systemic therapy for melanoma: ASCO guideline. J Clin Oncol 2020;38:3947-3970.

53. Uthurriague C, Thellier S, Ribes D, Rostaing L, Paul C, Meyer N. Vemurafenib significantly decreases glomerular filtration rate. $J$ Eur Acad Dermatol Venereol 2014;28:978-979.

54. Hurabielle C, Pillebout E, Stehlé T, et al. Mechanisms underpinning increased plasma creatinine levels in patients receiving vemurafenib for advanced melanoma. PLoS One 2016; 11:e0149873.

55. Launay-Vacher V, Zimner-Rapuch S, Poulalhon N, et al. Acute renal failure associated with the new BRAF inhibitor vemurafenib: a case series of 8 patients. Cancer 2014;120:2158-2163.

56. Jhaveri KD, Sakhiya V, Fishbane S. Nephrotoxicity of the BRAF inhibitors vemurafenib and dabrafenib. JAMA Oncol 2015;1:11331134.

57. Chiang AC, Herbst RS. Frontline immunotherapy for NSCLC the tale of the tail. Nat Rev Clin Oncol 2020;17:73-74.

58. Kennedy LB, Salama AKS. A review of cancer immunotherapy toxicity. CA Cancer J Clin 2020;70:86-104.

59. Almutairi AR, McBride A, Slack M, Erstad BL, Abraham I. Potential immune-related adverse events associated with monotherapy and combination therapy of ipilimumab, nivolumab, and pembrolizumab for advanced melanoma: a systematic review and meta-analysis. Front Oncol 2020;10:91.

60. Perazella MA, Shirali AC. Immune checkpoint inhibitor nephrotoxicity: what do we know and what should we do? Kidney Int 2020;97:62-74.

61. Manohar S, Kompotiatis P, Thongprayoon C, et al. Programmed cell death protein 1 inhibitor treatment is associated with acute kidney injury and hypocalcemia: meta-analysis. Nephrol Dial Transplant 2019;34:108-117.

62. Weber JS, Hodi FS, Wolchok JD, et al. Safety profile of nivolumab monotherapy: a pooled analysis of patients with advanced melanoma. J Clin Oncol 2017;35:785-792.

63. Cortazar FB, Marrone KA, Troxell ML, et al. Clinicopathological features of acute kidney injury associated with immune checkpoint inhibitors. Kidney Int 2016;90:638-647.

64. Wanchoo R, Karam S, Uppal NN, et al. Adverse renal effects of immune checkpoint inhibitors: a narrative review. Am J Nephrol 2017;45:160-169.

65. Seethapathy H, Zhao S, Chute DF, et al. The incidence, causes, and risk factors of acute kidney injury in patients receiving 
immune checkpoint inhibitors. Clin J Am Soc Nephrol 2019; 14:1692-1700.

66. Cortazar FB, Kibbelaar ZA, Glezerman IG, et al. Clinical features and outcomes of immune checkpoint inhibitor-associated AKI: a multicenter study. J Am Soc Nephrol 2020;31:435-446.

67. Shirali AC, Perazella MA, Gettinger S. Association of acute interstitial nephritis with programmed cell death 1 inhibitor therapy in lung cancer patients. Am J Kidney Dis 2016;68:287-291.

68. Kidd JM, Gizaw AB. Ipilimumab-associated minimal-change disease. Kidney Int 2016;89:720.

69. Fadel F, El Karoui K, Knebelmann B. Anti-CTLA4 antibody-induced lupus nephritis. N Engl J Med 2009;361:211-212.

70. Jung K, Zeng X, Bilusic M. Nivolumab-associated acute glomerulonephritis: a case report and literature review. BMC Nephrol 2016;17:188.

71. Perazella MA, Shirali AC. Nephrotoxicity of cancer immunotherapies: past, present and future. J Am Soc Nephrol 2018;29:20392052.

72. Brahmer JR, Lacchetti C, Schneider BJ, et al. Management of immune-related adverse events in patients treated with immune checkpoint inhibitor therapy: American Society of Clinical Oncology clinical practice guideline. J Clin Oncol 2018;36:17141768.

73. Haanen JB, Carbonnel F, Robert C, et al. Management of toxicities from immunotherapy: ESMO Clinical Practice Guidelines for diagnosis, treatment and follow-up. Ann Oncol 2017;28(Suppl 4):iv119-iv142.

74. Manohar S, Thongprayoon C, Cheungpasitporn W, Markovic SN, Herrmann SM. Systematic review of the safety of immune checkpoint inhibitors among kidney transplant patients. Kidney Int Rep 2019;5:149-158.

75. Murakami N, Riella LV. Co-inhibitory pathways and their importance in immune regulation. Transplantation 2014;98:3-14.

76. Abdel-Wahab N, Safa H, Abudayyeh A, et al. Checkpoint inhibi- tor therapy for cancer in solid organ transplantation recipients: an institutional experience and a systematic review of the literature. J Immunother Cancer 2019;7:106.

77. Moreau A, Varey E, Anegon I, Cuturi MC. Effector mechanisms of rejection. Cold Spring Harb Perspect Med 2013;3:a015461.

78. Tanaka K, Albin MJ, Yuan X, et al. PDL1 is required for peripheral transplantation tolerance and protection from chronic allograft rejection. J Immunol 2007; 179:5204-5210.

79. Hu B, Yang XB, Sang XT. Liver graft rejection following immune checkpoint inhibitors treatment: a review. Med Oncol 2019;36:94.

80. Kumar V, Shinagare AB, Rennke HG, et al. The safety and efficacy of checkpoint inhibitors in transplant recipients: a case series and systematic review of literature. Oncologist 2020;25:505-514.

81. Berraondo P, Sanmamed MF, Ochoa MC, et al. Cytokines in clinical cancer immunotherapy. Br J Cancer 2019;120:6-15.

82. Anders HJ, Lichtnekert J, Allam R. Interferon-alpha and -beta in kidney inflammation. Kidney Int 2010;77:848-854.

83. Weiss K. Safety profile of interferon-alpha therapy. Semin Oncol 1998;25(1 Suppl 1):9-13.

84. Guleria AS, Yang JC, Topalian SL, et al. Renal dysfunction associated with the administration of high-dose interleukin-2 in 199 consecutive patients with metastatic melanoma or renal carcinoma. J Clin Oncol 1994;12:2714-2722.

85. Poust JC, Woolery JE, Green MR. Management of toxicities associated with high-dose interleukin-2 and biochemotherapy. Anticancer Drugs 2013;24:1-13.

86. Gutgarts V, Jain T, Zheng J, et al. Acute kidney injury after CAR-T cell therapy: low incidence and rapid recovery. Biol Blood Marrow Transplant 2020;26:1071-1076.

87. Gupta S, Seethapathy H, Strohbehn IA, et al. Acute kidney injury and electrolyte abnormalities after chimeric antigen receptor T-cell (CAR-T) therapy for diffuse large B-cell lymphoma. Am J Kidney Dis 2020;76:63-71. 